\title{
Magnetic properties of ultrathin $3 d$ transition-metal binary alloys. I. Spin and orbital moments, anisotropy, and confirmation of Slater-Pauling behavior
}

\author{
Martin A. W. Schoen,,${ }^{1,2,}$ Juriaan Lucassen, ${ }^{3}$ Hans T. Nembach, ${ }^{1}$ T. J. Silva, ${ }^{1}$ Bert Koopmans, ${ }^{3}$ \\ Christian H. Back, ${ }^{2}$ and Justin M. Shaw ${ }^{1}$ \\ ${ }^{1}$ Quantum Electromagnetics Division, National Institute of Standards and Technology, Boulder, Colorado 80305, USA \\ ${ }^{2}$ Institute of Experimental and Applied Physics, University of Regensburg, 93053 Regensburg, Germany \\ ${ }^{3}$ Department of Applied Physics, Eindhoven University of Technology, 5600 MB, Eindhoven, The Netherlands
}

(Received 31 January 2017; published 7 April 2017)

\begin{abstract}
The structure and static magnetic properties—saturation magnetization, perpendicular anisotropy, spectroscopic $g$ factor, and orbital magnetization—of thin-film $3 d$ transition metal alloys are determined over the full range of alloy compositions via x-ray diffraction, magnetometry, and ferromagnetic resonance measurements. We determine the interfacial perpendicular magnetic anisotropy by use of samples sets with varying thickness for specific alloy concentrations. The results agree with prior published data and theoretical predictions. They provide a comprehensive compilation of the magnetic properties of thin-film $\mathrm{Ni}_{x} \mathrm{Co}_{1-x}, \mathrm{Ni}_{x} \mathrm{Fe}_{1-x}$, and $\mathrm{Co}_{x} \mathrm{Fe}_{1-x}$ alloys that goes well beyond the often-cited Slater-Pauling dependence of magnetic moment on alloy concentration.
\end{abstract}

DOI: $10.1103 /$ PhysRevB.95.134410

\section{INTRODUCTION}

The magnetic moment of binary $3 d$ transition metal alloys has been successfully described by the Slater-Pauling model $[1,2]$. This description is based on a rigid-band model where alloying of a $3 d$ transition metal ferromagnet with another element shifts the Fermi energy, and therefore, the occupation of the magnetic $d$ states around the Fermi energy. This occupation shift directly translates into a change in magnetization, leading to the basic triangular shape of the Slater-Pauling curve [3-5]. In the $\mathrm{Ni}_{x} \mathrm{Fe}_{1-x}$ and $\mathrm{Co}_{x} \mathrm{Fe}_{1-x}$ alloy systems, transitions from a body-centered-cubic (bcc) to a face-centered-cubic (fcc) phase are present. Such phase transitions result in a nontrivial change in the electronic band structure, which can be seen as additional features in the Slater-Pauling curve. For example, a local minimum of magnetic moment typically occurs in the $\mathrm{Ni}_{x} \mathrm{Fe}_{1-x}$ and $\mathrm{Co}_{x} \mathrm{Fe}_{1-x}$ alloys at the phase transition.

Despite the fact that the rigid band assumption does not strictly hold for most materials, the Slater-Pauling model [6,7] remains an effective tool for estimating the general behavior of $3 d$ transition metals. Indeed, further refinement of theoretical descriptions of alloy systems requires that the microscopic band structure is known or calculated, since alloying influences the band structure, for example by smearing out the bands due to atomic disorder [8]. Furthermore, other magnetic properties like orbital magnetization or magnetocrystalline anisotropy cannot be described semiclassically and are purely quantum mechanical in origin [9].

The development of new magnetic devices such as spintransfer-torque random-access memory (STT-RAM) [10,11] or spin-torque oscillators (STOs), requires the magnetic properties of each layer to be precisely tuned according to the specifics of the application. The binary alloys of $\mathrm{Ni}_{x} \mathrm{Co}_{1-x}$, $\mathrm{Ni}_{x} \mathrm{Fe}_{1-x}$, and $\mathrm{Co}_{x} \mathrm{Fe}_{1-x}$ exhibit a wide range of magnetic properties that were thoroughly investigated in bulk samples during the 1960s and 1970s [12-14]. However, many emerging technologies require films of these materials as thin as a

*Corresponding author: martin1.schoen@physik.uni-regensburg.de few monolayers. Such thin films can exhibit substantially modified magnetic properties from the bulk, including interfacial anisotropy [15,16], strain-induced anisotropy [17-19], reduction of the Curie temperature [20,21], or modification of the magnetic moment [22]. While there have been a number of investigations of materials as thin films [23-28], there is not yet a comprehensive and systematic study of thin $3 d$ transition metal alloy films that makes use of modern high-precision characterization methods-such as broad-band ferromagnetic resonance (FMR) or high-resolution $\mathrm{x}$-ray diffraction (XRD). A precise measurement of the magnetic properties for this relatively simple alloy system will facilitate their use in devices, as well as the development of new alloy systems. Here we report measurements of the alloys $\mathrm{Ni}_{x} \mathrm{Co}_{1-x}, \mathrm{Ni}_{x} \mathrm{Fe}_{1-x}$, and $\mathrm{Co}_{x} \mathrm{Fe}_{1-x}$ over the full range of compositions. We determined the in-plane lattice constant and crystalline structure via XRD, the saturation magnetization density $M_{S}$ via superconducting quantum interference device (SQUID) magnetometry, as well as the perpendicular anisotropy and the interfacial orbital magnetic moment via FMR. The data presented here are not only a glossary of high-precision measurements of the thin-film magnetic properties in $\mathrm{Ni}_{x} \mathrm{Co}_{1-x}, \mathrm{Ni}_{x} \mathrm{Fe}_{1-x}$, and $\mathrm{Co}_{x} \mathrm{Fe}_{1-x}$, but will also facilitate future testing of predictions based on theoretical calculations, e.g., density functional theory.

\section{SAMPLES AND METHOD}

Thin film samples consisting of $\mathrm{Ni}_{x} \mathrm{Co}_{1-x}, \mathrm{Ni}_{x} \mathrm{Fe}_{1-x}$, and $\mathrm{Co}_{x} \mathrm{Fe}_{1-x}$ alloys were grown at room temperature via $\mathrm{dc}$ magnetron sputter deposition on thermally oxidized (001) $\mathrm{Si}$ substrates at an Ar pressure of $0.67 \mathrm{~Pa}\left(5 \times 10^{-3}\right.$ Torr $)$. Substrates were kept in contact with a thermal reservoir to prevent substrate heating during the deposition process. Film compositions span the full range from $x=0$ to $x=1$. The sputter chamber had a base pressure of less than $5 \times 10^{-6} \mathrm{~Pa}\left(4 \times 10^{-8}\right.$ Torr $)$. A Ta(3 nm $) / \mathrm{Cu}(3 \mathrm{~nm})$ seed layer and $\mathrm{Cu}(3 \mathrm{~nm}) / \mathrm{Ta}(3 \mathrm{~nm})$ cap layer was used for all samples. The seed layer was chosen to maintain good adhesion to 
the substrate and promote a high quality textured crystalline structure. The capping layer prevents oxidation of the alloy layer, and provides approximately symmetric interfaces and boundary conditions for the excited magnetization. The alloys were co-sputtered from two targets with the deposition rates determined by $\mathrm{x}$-ray reflectivity (XRR). Drift in the deposition rates was periodically monitored with XRR and the repeatability of the deposition rates was found to be better than $3 \%$ over the course of the study. For all deposited alloys, the combined deposition rate was kept at approximately $0.25 \mathrm{~nm} / \mathrm{s}$ to ensure similar growth conditions. In order to quantitatively account for interfacial effects, we also deposited a thickness series that typically included 10, 7, 4, 3, and $2 \mathrm{~nm}$ thicknesses of the pure elements, as well as selected intermediate alloy concentrations $\left(\mathrm{Ni}_{63} \mathrm{Co}_{37}, \mathrm{Ni}_{20} \mathrm{Fe}_{80}, \mathrm{Ni}_{50} \mathrm{Fe}_{50}, \mathrm{Co}_{85} \mathrm{Fe}_{15}, \mathrm{Co}_{50} \mathrm{Fe}_{50}, \mathrm{Co}_{25} \mathrm{Fe}_{75}\right.$, and $\mathrm{Co}_{20} \mathrm{Fe}_{80}$ ). Following deposition, the samples were coated with $\approx 150 \mathrm{~nm}$ poly(methyl methacrylate) (PMMA) for both mechanical protection and to prevent direct electrical contact to the co-planar waveguide (CPW) used for broadband FMR measurements.

Broadband FMR characterization in the out-of-plane geometry was performed by use of a room temperature bore superconducting magnet capable of applying a perpendicular external magnetic field $H$ as large as $\mu_{0} H=3 \mathrm{~T}$. Samples were placed face-down on a CPW with a center conductor width of $100 \mu \mathrm{m}$ with a nominal impedance of $50 \Omega$. A vector network analyzer (VNA) was connected to both ends of the $\mathrm{CPW}$, and the complex $S_{21}$ transmission parameter (ratio of voltage applied at one end of the CPW to voltage measured at the other end) was measured over a frequency range of 10 to $40 \mathrm{GHz}$. $S_{21}$ was then fitted with the complex susceptibility tensor component $\chi_{z z}$. For the purpose of fitting, we use

$$
S_{21}(H)=A \chi_{z z}(H) e^{i \phi}+m H,
$$

with the phase $\phi$ and the dimensionless mode amplitude $A$. A field-dependent complex linear background $m H$ was subtracted to account for measurement drifts. The susceptibility component is derived from the Landau-Lifshitz equation for the perpendicular geometry ( $z$ axis). In the fixed-frequency, field-swept configuration we obtain [29]

$$
\chi_{z z}(H)=\frac{M_{S}\left(H-M_{\mathrm{eff}}\right)}{\left(H-M_{\mathrm{eff}}\right)^{2}-\left(H_{\mathrm{eff}}\right)^{2}-i \Delta H\left(H-M_{\mathrm{eff}}\right)},
$$

where $M_{\text {eff }}=M_{S}-H_{k}$ is the effective magnetization, $M_{S}$ is the saturation magnetization, $H_{k}$ is the perpendicular anisotropy, and $\Delta H$ is the linewidth. $H_{\text {eff }}=2 \pi f /\left(\gamma \mu_{0}\right)$, where $|\gamma|$ is the gyromagnetic ratio and $\mu_{0}$ is the vacuum permeability. An example of the measured FMR spectra is plotted in Fig. 1, where we present both the real and imaginary parts of $S_{21}$ for $\mathrm{Ni}_{90} \mathrm{Fe}_{10}$ measured at $20 \mathrm{GHz}$, in addition to the susceptibility fit to the data.

Both the effective magnetic field $M_{\text {eff }}$ and the spectroscopic $g$ factor were determined from the resonance field $H_{\text {res }}$ vs frequency $f$ plot [compare Fig. 1 (c)] according to

$$
H_{\text {res }}=M_{\text {eff }}+\frac{h}{g \mu_{B \mu_{0}}} f,
$$

where $\mu_{\mathrm{B}}$ is the Bohr-magneton. The extracted values for $g$ and $M_{\text {eff }}$ are then corrected for errors stemming from the limited
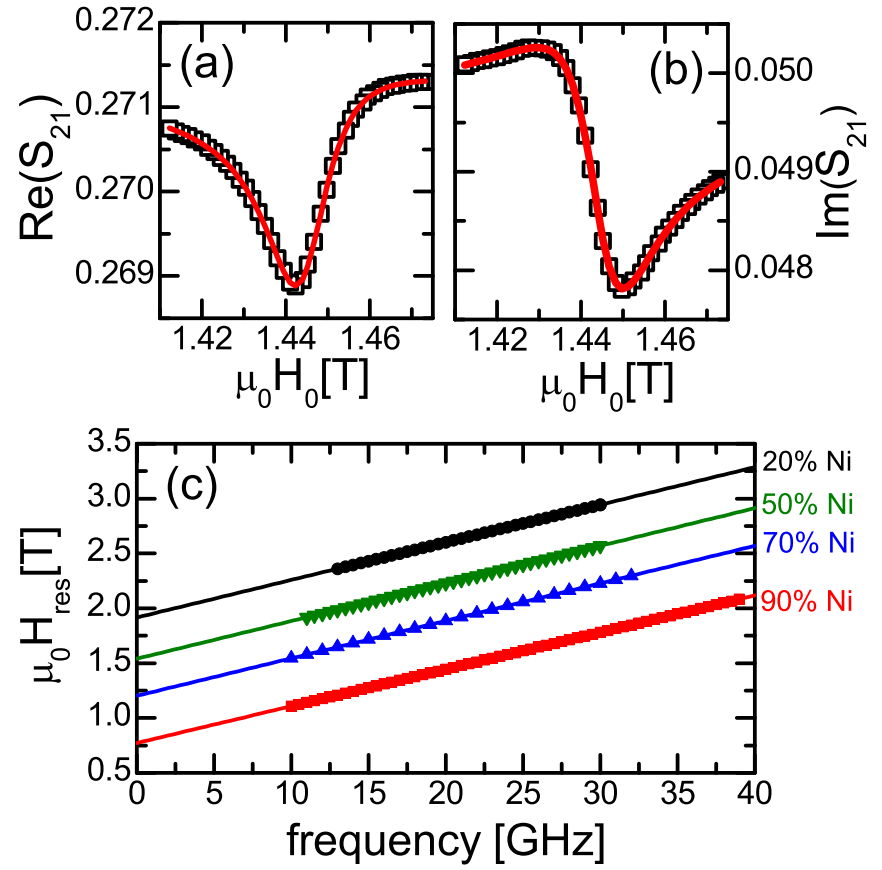

FIG. 1. (a) and (b), respectively, show the real and imaginary part of the $S_{21}$ transmission parameter (black squares) measured at $20 \mathrm{GHz}$, along with the complex susceptibility fit (red lines) for the $10 \mathrm{~nm} \mathrm{Ni} \mathrm{N}_{90} \mathrm{Fe}_{10}$ alloy. In (c), the resonance fields of four $\mathrm{Ni}_{x} \mathrm{Fe}_{1-x}$ alloys are plotted against the frequency (data points) and fitted linearly (lines) with $\mathrm{Ni}$ concentrations denoted on the right axis. The zero-frequency intercepts of the linear fits determine the effective magnetization and their slope is inversely proportional to the $g$ factor.

measured frequency range via the method described by Shaw et al. [30].

The crystal structure was characterized by in-plane x-ray diffraction (XRD) using parallel beam optics with a $\mathrm{Cu} K_{\alpha}$ radiation source.

\section{RESULTS}

\section{A. XRD}

Figure 2 shows the in-plane lattice constants, determined from the [211] bcc and [111] fcc peaks in the diffraction spectra. The $\mathrm{Cu}(220)$ peak is always visible in the spectra due to the $\mathrm{Cu}$ seed and cap layers used in all the samples. This complicates the analysis since the location of the fccbcc phase transition for the $\mathrm{Ni}_{x} \mathrm{Fe}_{1-x}$ and $\mathrm{Co}_{x} \mathrm{Fe}_{1-x}$ alloys cannot be determined exactly. However, the change in the fcc lattice constant away from the bulk $\mathrm{Cu}$ value, as well as the diminishment and disappearance of the observed bcc peak, allows us to determine a concentration window in which a mixed phase occurs near the phase transition. Furthermore, we determined the texture of the bcc and fcc phases to be (110) and (111), respectively.

The $\mathrm{Ni}_{x} \mathrm{Fe}_{1-x}$ alloys exhibit a bcc phase and unchanging bcc lattice constant for a $\mathrm{Ni}$ concentration between $0 \%$ and $20 \%$. For Ni concentrations between $20 \%$ and $30 \%$ a mixed phase [3] is measured as determined by the change in the fcc lattice constant to lower values than for fcc $\mathrm{Cu}$, indicating the formation of a fcc phase in coexistence with a bcc phase, 

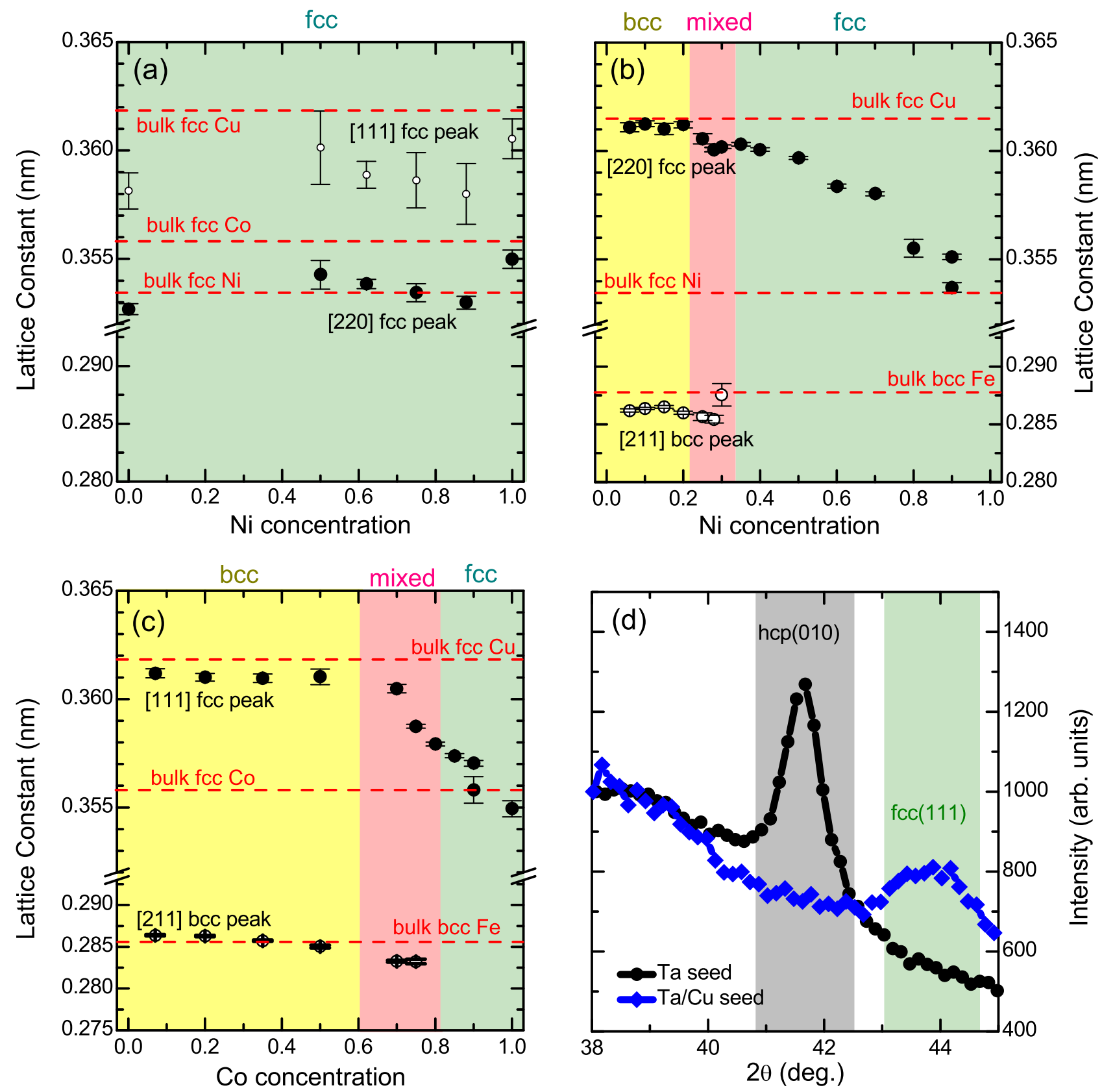

FIG. 2. The in-plane lattice constants for (a) $\mathrm{Ni}_{x} \mathrm{Co}_{1-x}$, (b) $\mathrm{Ni}_{x} \mathrm{Fe}_{1-x}$, and (c) $\mathrm{Co}_{x} \mathrm{Fe}_{1-x}$, as determined by XRD, where the closed circles signify the lattice constant determined from the [220] fcc peak and the open circles either signify the lattice constant from the [111] fcc peak in (a), or from the [211] bcc peak in (b) and (c). Note that the fcc lattice constants are always determined from a superposition of the Cu XRD signal and the fcc alloy XRD signal. The interpretation of multiple peaks is discussed in Sec. III A. (d) XRD spectra for pure Co grown on both a Ta seed layer and a Ta/Cu seed layer. The Co grown on Ta shows a clear hcp peak, while that grown on Ta/Cu exhibits only an fcc peak.

as seen in Fig. 2(b). The location of this transition is also consistent with the concentration previously reported in the bulk $\mathrm{Ni}_{x} \mathrm{Fe}_{1-x}$ system at $30 \% \mathrm{Ni}$ [3]. For Ni concentrations above $30 \%$ the $\mathrm{Ni}_{x} \mathrm{Fe}_{1-x}$ alloys exhibit a pure fcc phase, with a lattice constant that approaches the value for pure bulk $\mathrm{Ni}$ as the $\mathrm{Ni}$ concentration increases.

The $\mathrm{Co}_{x} \mathrm{Fe}_{1-x}$ system also exhibits a similar fcc-bcc phase transition. The alloys are bcc up to a Co concentration of $60 \%$, followed by an fcc to bcc phase transition in the vicinity of $70 \%$ Co. This phase transition is again confirmed by XRD, but for the same reason as for the $\mathrm{Ni}_{x} \mathrm{Fe}_{1-x}$ system, we could only determine that the exact location of the bcc to fcc phase transition occurs somewhere between $60 \%$ and $80 \%$ Co, where bcc and fcc phases coexist, as shown in Fig. 2(c). This phase transition seems to occur at a lower Co concentration than for the bulk alloy system [3], which can be attributed to the $\mathrm{Cu}$ seed layer, as elaborated in the next paragraph [33]. Above $80 \%$ Co the $\mathrm{Co}_{x} \mathrm{Fe}_{1-x}$ alloys exhibit purely fcc phase.

Our pure Co films do not exhibit a hexagonal close-packed (hcp) crystal structure, in contrast to prior reports in the literature [3]. Figure 2(d) shows the XRD spectrum in the vicinity of the $\mathrm{hcp}(010)$ peak for the $10 \mathrm{~nm}$ pure Co sample, 
with a $\mathrm{Ta} / \mathrm{Cu}$ seed and a $\mathrm{Cu} / \mathrm{Ta}$ cap layer. For comparison, we include a similar Co film that was grown with only Ta as seed and capping layers. The sample with only Ta in the seed exhibits a clear hcp(010) peak, indicating an hcp structure. In contrast, the sample that includes $\mathrm{Cu}$ in the seed and capping layers shows no evidence of hcp structure. We speculate that the strained growth of $\mathrm{Co}$ on $\mathrm{Cu}$ promotes a strained fcc (i.e., face-centered tetragonal, fct) phase in the Co [31], which is consistent with the structure reported for room-temperature growth of $\mathrm{Co} / \mathrm{Cu}$ layers via molecular epitaxy [31-33].

The crystalline phase of the $\mathrm{Ni}_{x} \mathrm{Co}_{1-x}$ alloys is exclusively fcc and exhibits distinguishable fcc(111) and fcc(220) peaks. The first peak, with its larger lattice constant, can be attributed to $\mathrm{Cu}$, while we attribute the second peak to the $\mathrm{Ni}_{x} \mathrm{Co}_{1-x}$.

\section{B. Magnetization}

We determined the room temperature (RT) saturation magnetization $M_{S}$ for all samples via superconducting quantum interference device (SQUID) magnetometry. The samples were first diced with a precision diamond saw such that the surface area of the sample is accurately known. The saturation magnetization $M_{S}$ is then determined by dividing the measured magnetic moment by the volume of the magnetic layer. The sample volumes were corrected to account for interfacial factors, like the existence of a dead layer or alloying with the $\mathrm{Cu}$ cap and seed layers. Such interfacial effects on the magnetization are determined in a thickness series for select alloys, measured with FMR. The $x$ intercept of the $M_{\text {eff }}$ vs $1 / t$ plot (not shown) show a nonzero value of $1.4 \mathrm{~nm}^{-1}$, indicating the existence of either a $(0.7 \pm 0.3) \mathrm{nm}$ magnetic dead layer, or a layer of reduced magnetization corresponding to a thickness of approximately two monolayers on each interface for all measured samples. The thicknesses of the dead layers display no discernible trend for alloy composition. The existence of a dead layer of this thickness has been found for $\mathrm{Fe}$ and $\mathrm{Ni}_{80} \mathrm{Fe}_{20}$ at similar interfaces [34,35]. Adjusted for the $0.7 \mathrm{~nm}$ dead layer, the SQUID measurements were normalized to the alloy volume and the resulting $M_{S}$ for all alloys is plotted in Figs. 3(a)-3(c) (blue triangles).

For the $\mathrm{Ni}_{x} \mathrm{Co}_{1-x}$ alloys, $M_{S}$ decreases almost linearly with increasing $\mathrm{Ni}$ concentration from $\mu_{0} M_{S}(\mathrm{Co})=$ $(1.77 \pm 0.04) \mathrm{T}$ to $\mu_{0} M_{S}(\mathrm{Ni})=(0.51 \pm 0.03) \mathrm{T}$. In the $\mathrm{Ni}_{x} \mathrm{Fe}_{1-x}$ alloy system $M_{S}$ increases from $\mu_{0} M_{S}(\mathrm{Fe})=$ $(2.05 \pm 0.02) \mathrm{T}$ to a maximum of $(2.12 \pm 0.06) \mathrm{T}$ at $10 \%$ $\mathrm{Ni}$ followed by a minimum at the phase transition $(25 \% \mathrm{Ni})$. At $\mathrm{Ni}$ concentrations greater than $40 \% M_{S}$ decreases again with increasing $\mathrm{Ni}$ concentration. $M_{S}$ of the $\mathrm{Co}_{x} \mathrm{Fe}_{1-x}$ alloys shows a maximum of $(2.42 \pm 0.05) \mathrm{T}$ at approximately $35 \%$ Co followed by a decrease with higher Co concentration and a drop at the phase transition.

This behavior for $M_{S}$ is consistent with the often-observed Slater-Pauling curve $[1,2,36]$, which is included in Fig. 3 as the gray dotted lines. The only deviations occur in the vicinity of the $\mathrm{Ni}_{x} \mathrm{Fe}_{1-x}$ and $\mathrm{Co}_{x} \mathrm{Fe}_{1-x}$ phase transitions. In those cases, the "dip" or "drop" in the curve occur at lower Ni or, respectively, Co concentration than in the bulk Slater-Pauling curve. This is consistent with our XRD measurements that indicate promotion of the fcc phase by the $\mathrm{Cu}$ substrate, which causes small deviations relative to the bulk phase diagram.
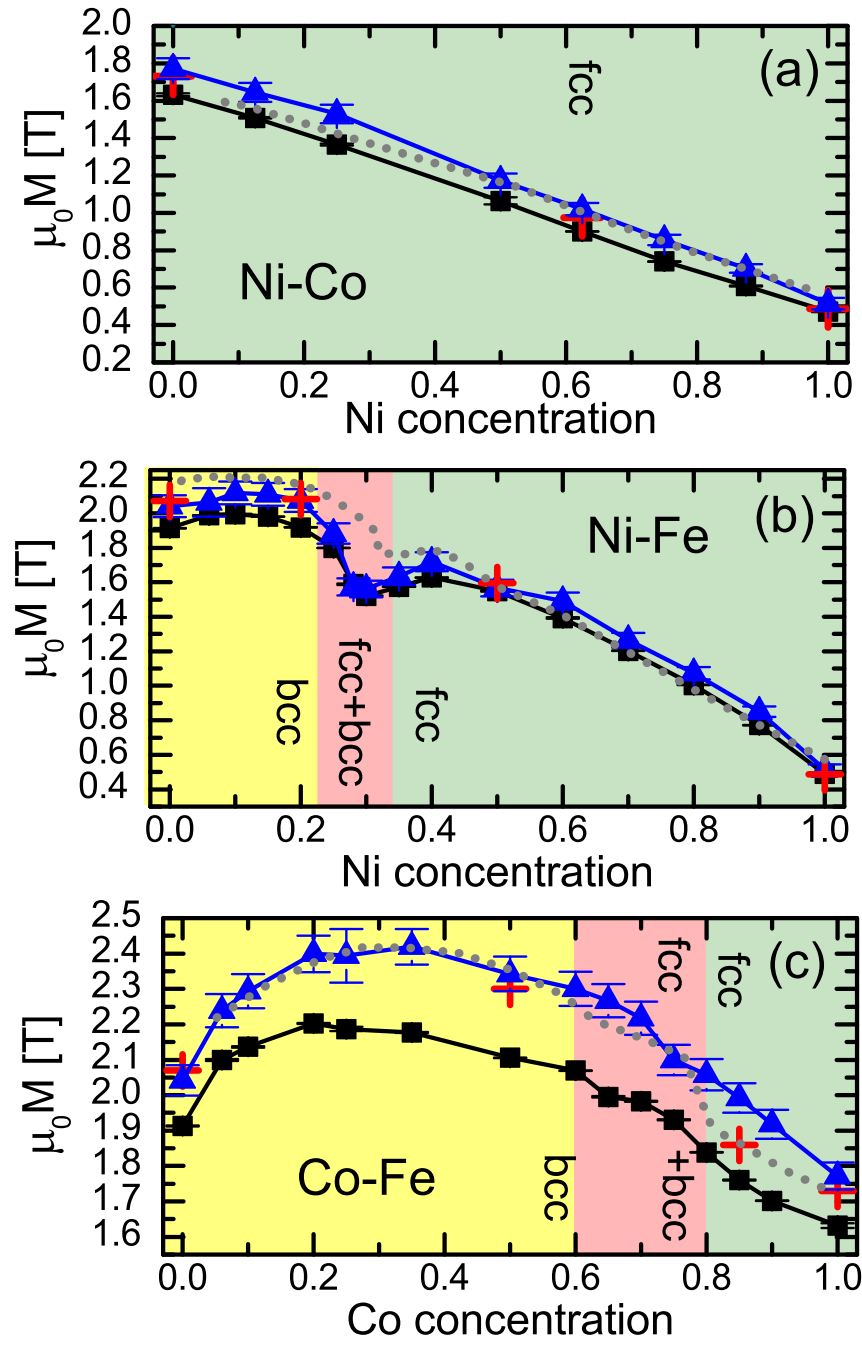

FIG. 3. Room temperature effective magnetization $M_{\text {eff }}$ (black squares), measured via FMR, and the room temperature saturation magnetization $M_{S}$ (blue triangles), measured via SQUID magnetometry, are plotted in (a) for $\mathrm{Ni}_{x} \mathrm{Co}_{1-x}$, in (b) for $\mathrm{Ni}_{x} \mathrm{Fe}_{1-x}$ and in (c) for $\mathrm{Co}_{x} \mathrm{Fe}_{1-x}$. For comparison, $M_{S}$ is also determined by linear regression of $M_{\text {eff }}$ vs $1 / t$ (red crosses). They match $M_{S}$ by SQUID at those alloy concentrations reasonably well. This shows that the SQUID and FMR measurements are consistent. The crystal structure of the alloys is denoted and signified in the same color code as in Fig. 2. For comparison the bulk "Slater-Pauling" curves for the three alloy systems are also plotted (gray dotted lines) [3].

The effective magnetization $M_{\text {eff }}$ is determined by use of Eq. (3) to analyze the FMR data. With the assumption of purely interfacial perpendicular anisotropy, i.e., negligible bulk perpendicular anisotropy, the saturation magnetization $M_{S}$ can also be determined by measuring $M_{\text {eff }}$ in a thickness series $(10,7,4,3$, and $2 \mathrm{~nm})$ and taking the $y$ intercept (corresponding to infinite thickness $t$ ) when $M_{\text {eff }}$ is plotted versus $1 / t$. This is done for a select number of alloys and the resulting values of $M_{S}$ determined from FMR are also included in Fig. 3 (red crosses). These values for $M_{S}$ agree well with the values of $M_{S}$ determined by SQUID, demonstrating the equivalence of both measurement methods. 
Furthermore, we determine $M_{\text {eff }}$ for the 10-nm-thick alloy samples for all concentrations via Eq. (3), with the results plotted in Figs. 3(a)-3(c) (black squares). $M_{\text {eff }}$ generally follows the Slater-Pauling curve with an offset due to the presence of interfacial perpendicular anisotropy.

\section{Perpendicular magnetic anisotropy}

As already stated, the difference between $M_{\text {eff }}$ from FMR and $M_{S}$ from magnetometry is the result of interfacial perpendicular anisotropy $H_{k}$, which results from the broken symmetry at the interfaces [37,38]. Despite being purely interfacial, this interface anisotropy acts on the whole film in the thin film limit and is therefore often employed to engineer the anisotropy according to certain specifications, in particular for perpendicularly magnetized materials [39-42]. Defining the anisotropy energy as in Ref. [43], the effective total perpendicular anisotropy energy density $K$ can be determined from $M_{S}$ and $M_{\text {eff }}$ via [18] $K=1 / 2\left(M_{S}-M_{\text {eff }}\right) M_{S} \mu_{0}$, and is plotted as a function of alloy concentration in Fig. 4. Note here that we do not separate the different contributions to $K$ (second and fourth order anisotropy constants) [44].

For the $\mathrm{Ni}_{x} \mathrm{Co}_{1-x}$ alloy, $K$ decreases almost linearly from pure Co to pure Ni. For the $\mathrm{Ni}_{x} \mathrm{Fe}_{1-x}$ alloys, $K$ has a sharp minimum at the phase transition. We speculate that the minimum is due to the coexistence of multiple phases with compensating amounts of anisotropy. Then, with higher $\mathrm{Ni}$ content, $K$ decreases almost linearly.

The $\mathrm{Co}_{x} \mathrm{Fe}_{1-x}$ alloys behave in a very different manner. Thin films of pure $\mathrm{Co}$ and $\mathrm{Fe}$ exhibit similar anisotropies,
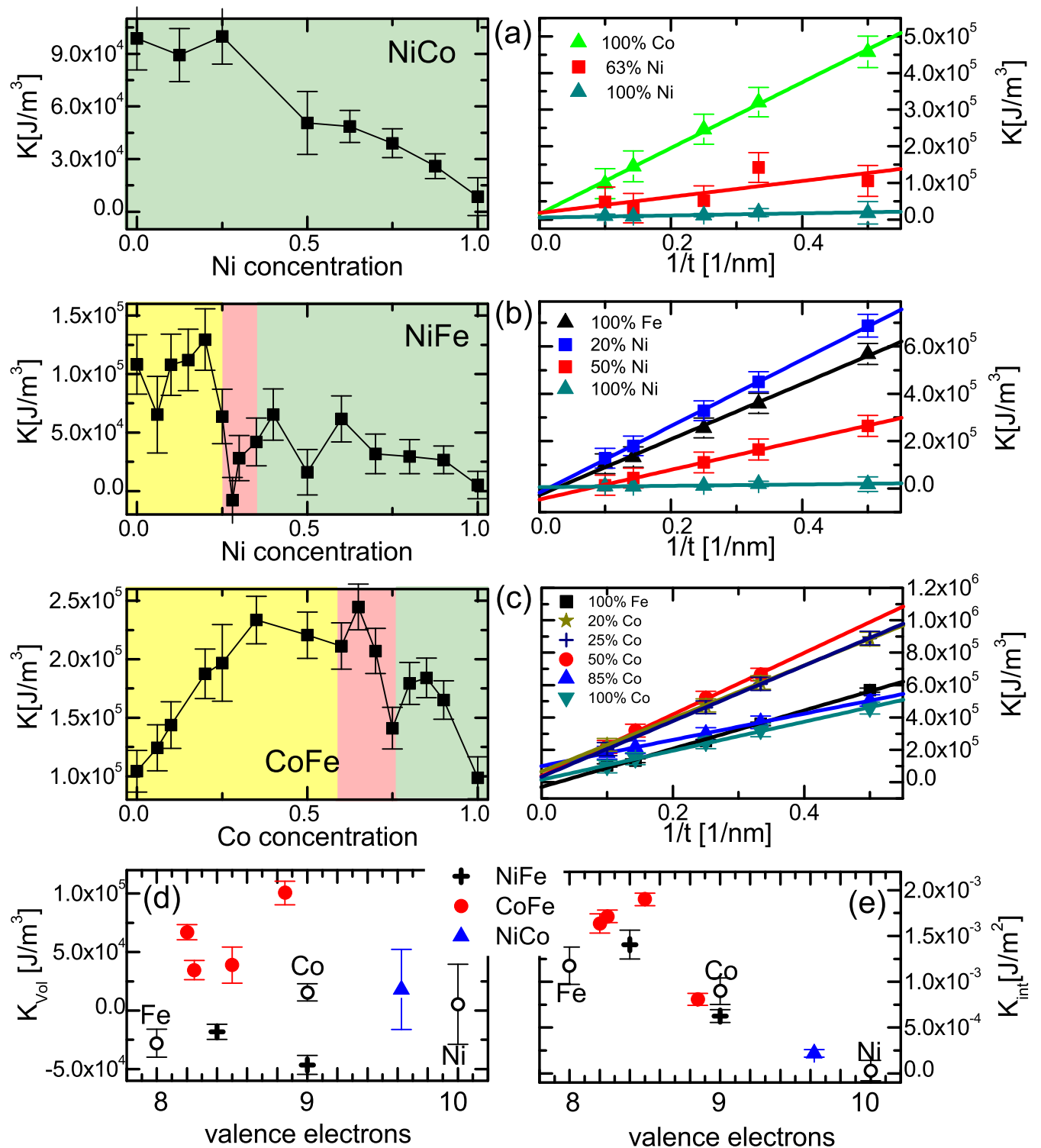

FIG. 4. The volume averaged perpendicular anisotropy energy density $K$ is plotted vs alloy composition for (a) $\mathrm{Ni}_{x} \mathrm{Co}_{1-x},(\mathrm{~b}) \mathrm{Ni}_{x} \mathrm{Fe}_{1-x}$, and (c) $\mathrm{Co}_{x} \mathrm{Fe}_{1-x}$. Its thickness dependence for select alloys is plotted in the adjoining panels to the right. Again the crystal structure is signified in color code. The perpendicular anisotropy shows local minimal where fcc and bcc phases coexist for both $\mathrm{Ni}_{x} \mathrm{Fe}_{1-x}$ and $\mathrm{Co}_{x} \mathrm{Fe}_{1-x}$. In (d) and (e) we, respectively, plot the volume averaged bulk anisotropy energy density $K_{\mathrm{vol}}$ and the total interfacial anisotropy for both FM/Cu interfaces $K_{\text {int }}$, extracted from the intercept and slope via linear regression of $K$ vs reciprocal thickness $1 / t$. 


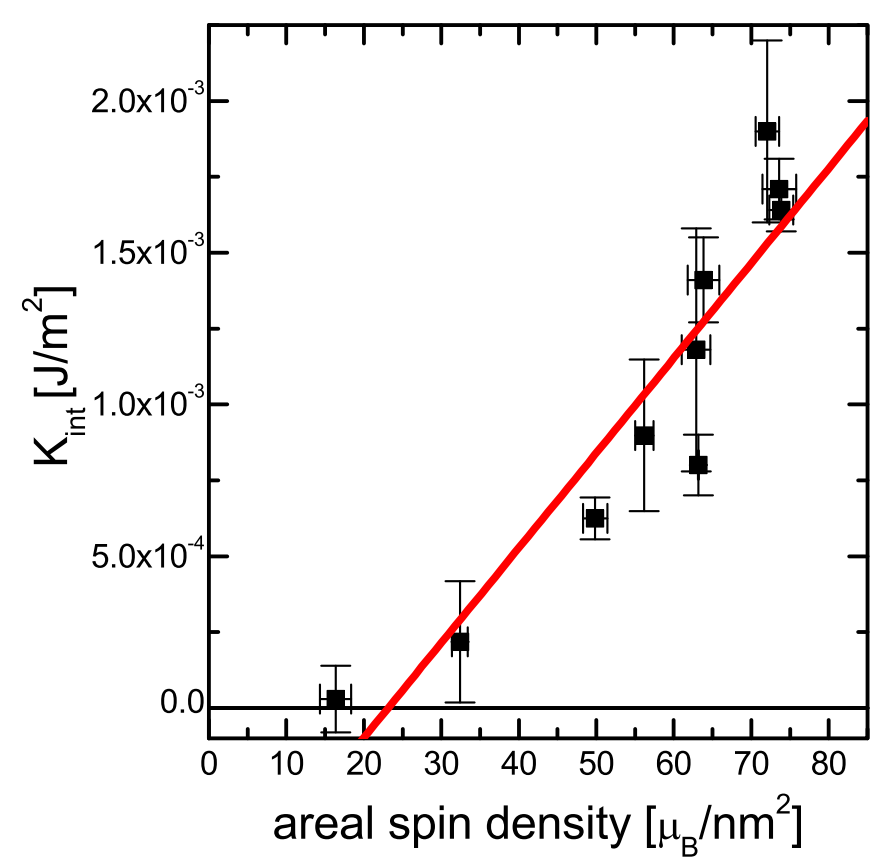

FIG. 5. The interfacial anisotropy $K_{\text {int }}$ is plotted against the areal spin density. The red line is a linear fit to the data.

but the alloys have higher values for the anisotropy, up to $2.3 \times 10^{5} \mathrm{~J} / \mathrm{m}^{3}$, as shown in Fig. 4(c). At a Co concentration of $75 \%$ near the fcc to bcc phase transition, $K$ exhibits some degree of distortion.

Under the assumption that $M_{S}$ is independent of thickness above $2 \mathrm{~nm}$ (e.g., $\mathrm{Cu}$ does not alloy with the magnetic films), the bulk anisotropy $K_{\mathrm{vol}}$ and the average interfacial anisotropy $K_{\text {int }}$ can be determined from the thickness dependence of the total anisotropy $K$ by use of the phenomenological equation $K(t)=K_{\mathrm{vol}}+2 K_{\mathrm{int}} / t$ (the factor of 2 accounts for the number of interfaces) [45], where fits of the data based on this equation are presented in the right panels in Fig. 4. We plot the volume and interface components of $K$ with respect to atomic number in Figs. 4(d) and 4(e). $K_{\mathrm{vol}}$ is small to negligible with no discernable trend with alloy composition. The interface components of the total perpendicular anisotropy for the $\mathrm{Co}_{33} \mathrm{Ni}_{67}$ alloy is in the range of the one reported by Shaw et al. [45] of $2 K_{\text {int }}=1.56 \times 10^{-4} \mathrm{~J} / \mathrm{m}^{2}$ for a $\left(\mathrm{Co}_{90} \mathrm{Fe}_{10}\right)_{25} \mathrm{Ni}_{75}$ alloy with the same seed and cap layers as used in this study.

Surprisingly, $K_{\text {int }}$ exhibits similar Slater-Pauling behavior as the magnetization data in Fig. 3. This behavior suggests that there is a certain amount of interfacial anisotropy energy per uncompensated $d$-band spin, i.e., the anisotropy energy is proportional to the spin density at the interface. In Fig. 5 we plot the interface anisotropy against areal spin density and indeed $K_{\text {int }}$ increases with the areal spin density. A linear fit to the data yields an $x$ intercept of $(23 \pm 7) \mu_{\mathrm{B}} / \mathrm{m}^{2}$, which translates to a magnetization of $(0.7 \pm 0.2) \mu_{\mathrm{B}}$ per interface atom. Considering the symmetry at the $3 d$ transition metal alloy/Cu interface a nonzero $x$ intercept seems reasonable. The symmetry of the localized alloy $d$ bands is largely broken at the interface with the mostly $s$-like $\mathrm{Cu}$ bands, while the alloy $s$ - $p$ band symmetry should be less affected $[46,47]$. Thus only the uncompensated localized $d$ bands should effectively contribute to the perpendicular anisotropy.

While the picture for the itinerant nature of magnetism in the $3 d$ metals remains incomplete, there is substantial evidence that the magnetization in $\mathrm{Ni}$ is not found solely in localized $d$ bands. Tunneling spectroscopy measurements of spin polarization of the $s$ - $p$-like conduction electrons for $\mathrm{Ni}$ have found values around $23 \%$ to $46 \%$ (compare Ref. [48] and references therein), which is close to the $x$ intercept value of the linear fit in Fig. 5. It is understood that the $s-p$ bands are strongly hybridized with the $d$ band near the Fermi surface, giving rise to a high degree of spin polarization for the conduction bands in Ni near the Fermi surface, as revealed by angle resolved photoemission spectroscopy (ARPES) [49]. On the other hand, the $d$ bands in $\mathrm{Fe}$ are believed to be more localized [5,50,51].These considerations side with the value of the $x$ intercept of the linear fit to the data, which is close to $M_{S}$ of Ni. Furthermore, we can estimate the perpendicular interfacial anisotropy energy per $d$-band spin to be $(2 \pm 0.6) \times 10^{-4} \mathrm{eV} / \mu_{B}$.

Note that the measured interface anisotropies are specifically for the $\mathrm{Cu} /$ alloy/Cu interfaces prepared for this study. It is very likely that these anisotropies will also vary with both the choice of nonmagnetic metal and the deposition conditions. It is also important to emphasize that the crystalline texture will affect the interfacial anisotropy [52]. In the present case, all of the fcc materials possess a (111) texture, whereas the bcc materials have a (110) texture. But the general trend may indicate a starting point in the search for alloy systems with the desired interface anisotropies.

\section{D. $g$ factor and orbital magnetization}

We now turn to the measured spectroscopic $g$ factor that describes the relationship between the spin angular momentum and total magnetic moment per electron.

For pure $\mathrm{Fe}$ and $\mathrm{Ni}$, the $g$ factors are $g_{\mathrm{Fe}}=2.085 \pm 0.003$ and $\mathrm{Ni} g_{\mathrm{Ni}}=2.182 \pm 0.016$, respectively. For comparison, previously reported values for bulk $\mathrm{Fe}$ and $\mathrm{Ni}$ are $\mathrm{g}_{\mathrm{Fe}}=2.08$ and $\mathrm{g}_{\mathrm{Ni}}=2.185[3,13,53]$, in good agreement with our results for 10-nm-thick films. The agreement between bulk and thin film values is not necessarily expected since there can be a substantial contribution of the orbital moment at the interface [45]. Similarly, the $g$ factor of Co is $g_{\mathrm{Co}}=2.139 \pm 0.005$, which is very close to the value previously reported for thin film fcc Co $g_{\text {Co }}=2.145$ [32]. This value is considerably smaller than the one for bulk hcp Co in literature of $\mathrm{g}_{\mathrm{Co}}=2.18$ $[3,13]$. This is consistent with the XRD results that show no evidence of an hcp phase for the pure Co film. Furthermore, the measured $g$ factor of Permalloy $\left(\mathrm{Ni}_{80} \mathrm{Fe}_{20}\right)$ is within $0.2 \%$ of the $g$ factor of $g_{\mathrm{Py}}=2.109$ previously reported by Shaw et al. [30]. We found that the $g$ factor decreases for most alloys with decreasing layer thickness, which has already been observed $[30,45]$. Interestingly the $g$ factor increases with decreasing thickness for pure $\mathrm{Co}$ and the $\mathrm{Co}_{50} \mathrm{Fe}_{50}$ alloy.

The $g$ factor for $\mathrm{Ni}_{x} \mathrm{Co}_{1-x}$ stays approximately constant for Ni concentrations between $12 \%$ and $66 \%$ after an initial increase from pure fcc Co. At Ni concentrations above $66 \%, g$ approaches the value of pure Ni. For comparison, the $g$ factor for hcp Co is also plotted in Figs. 6(a) and 6(c). Assuming 

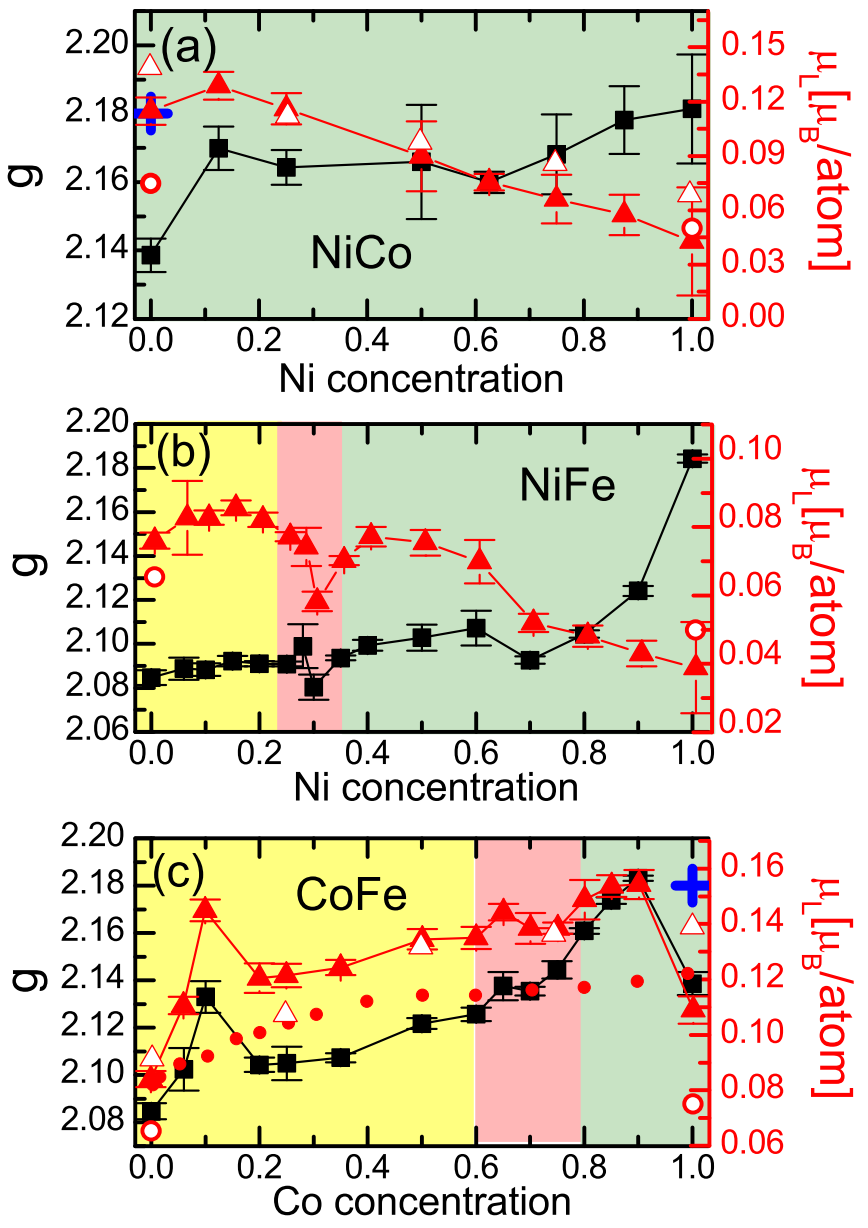

FIG. 6. The measured out-of-plane spectroscopic $g$ factor (black squares, left axis) and the orbital contribution to the saturation magnetization $\mu_{\mathrm{L}}$ (red triangles, right axis) are plotted for (a) $\mathrm{Ni}_{x} \mathrm{Co}_{1-x}$, (b) $\mathrm{Ni}_{x} \mathrm{Fe}_{1-x}$, and (c) $\mathrm{Co}_{x} \mathrm{Fe}_{1-x}$ against the respective alloy composition (crystal phases are again signified in color). In (a) and (c) the $g$ factor for pure hcp Co is added [3,13] (blue cross). Orbital moments for the pure elements calculated by Malashevich et al. [54] are plotted as red open circles for comparison. Also orbital moments for the $\mathrm{Ni}_{x} \mathrm{Co}_{1-x}$ and $\mathrm{Co}_{x} \mathrm{Fe}_{1-x}$ systems calculated by Söderlind $e t$ al. [55] (red open triangles) as well as the orbital moment calculated for $\mathrm{Co}_{x} \mathrm{Fe}_{1-x}$ by Chadov et al. [56] (red dots) are included in the respective panels.

pure hcp Co a constant $g$ factor of 2.17 is, within a $1 \%$ scatter, a good approximation for the $g$ factor of all $\mathrm{Ni}_{x} \mathrm{Co}_{1-x}$ alloys. The $\mathrm{Ni}_{x} \mathrm{Fe}_{1-x}$ alloys display a different behavior with $\mathrm{Ni}$ concentration. Starting from pure $\mathrm{Fe}$ to $\mathrm{Ni}_{80} \mathrm{Fe}_{20}, g$ only shows an incremental increase, followed by a strong increase in $g$ toward the value for pure $\mathrm{Ni}$. The $g$ factor in the $\mathrm{Co}_{x} \mathrm{Fe}_{1-x}$ alloys exhibits a strong nonmonotonic behavior. $g$ increases with $\mathrm{Co}$ concentration from the value for pure $\mathrm{Fe}$ and displays a maximum at $10 \%$ Co, followed by a minimum at approximately $20 \%$ Co. With higher Co concentration the alloy $g$ factor increases towards the value for hcp Co and only drops again for pure fcc Co.

We do not observe a strong variation of $g$ factor around the fcc-bcc phase transition of $\mathrm{Ni}_{x} \mathrm{Fe}_{1-x}$, contrary to the previous report by Bauer and Wigen [12]. Instead, our data for
$\mathrm{Ni}_{x} \mathrm{Fe}_{1-x}$ follow a similar trend as that reported by Meyer and Ash [13].

The orbital contribution to the magnetization can be calculated from the measured $g$ factor. As a result of the spin-orbit interaction, the $\mathrm{g}$ factor can differ significantly from its undressed value of $\approx 2$. The ratio of orbital $\mu_{\mathrm{L}}$ and spin $\mu_{\mathrm{s}}$ electron moments, to the total magnetic electron moment $\mu$ is given by [57]

$$
\frac{\mu_{\mathrm{L}}}{\mu_{\mathrm{s}}}=\frac{g-2}{2} .
$$

We use our SQUID magnetometry data to determine the total magnetic moment per atom for each alloy, where we make use of previously published data for alloy atom density [3]. This is used to calculate the spin and orbital moment per atom by use of Eq. (4). The atomic orbital moment in Bohr magnetons $\mu_{\mathrm{B}}$ is plotted in Fig. 6 (right axis, red triangles).

Our values agree well with the previous report for the $\mathrm{Ni}_{x} \mathrm{Co}_{1-x}$ alloys of Reck and Fry [14]. For $\mathrm{Ni}_{x} \mathrm{Fe}_{1-x}$, their reported $\mu_{\mathrm{L}}$ is slightly larger than our measured value. It was not possible to discern if the nonmonotonic feature exhibited by the $\mathrm{Co}_{x} \mathrm{Fe}_{1-x}$ alloys were also observed by Reck and Fry due to the density of data in the original report.

Our measurements are consistent with the well-known fact that $\mu_{\mathrm{L}}$ is generally small and on the order of a few percent of the total atomic moment in crystals with cubic symmetry. Beyond that qualitative comparison, the precision of our data enable us to test theoretical $a b$ initio models for orbital moments. Theoretical values for $\mu_{\mathrm{L}}$ for pure $\mathrm{Ni}, \mathrm{Fe}$, and Co [54] reported by Malashevich et al., are included in Fig. 6 as red open circles. For pure bcc Fe and hcp Co, the calculations yield values that are approximately $30 \%$ lower than our experimentally determined values, whereas for fcc $\mathrm{Ni}$ the theory values are larger than the experimental values of $\mu_{\mathrm{L}}$. The results of Söderlind et al. [55] for the $\mathrm{Ni}_{x} \mathrm{Co}_{1-x}$ and $\mathrm{Co}_{x} \mathrm{Fe}_{1-x}$ alloys are included as red open triangles, and the theoretical predictions of Chadov et al. [56] for the $\mathrm{Co}_{x} \mathrm{Fe}_{1-x}$ system are included as red dots. The predictions of Söderlind et al. for the $\mathrm{Ni}_{x} \mathrm{Co}_{1-x}$ and $\mathrm{Co}_{x} \mathrm{Fe}_{1-x}$ alloys are remarkably close to our measured values, with the possible exception of pure Co, where we measured $\mu_{\mathrm{L}}=0.11 \mu_{\mathrm{B}}$, and they predicted $0.14 \mu_{\mathrm{B}}$. The calculated results of Chadov et al. match our measured values for pure $\mathrm{Co}$ and Fe quite well, but they are significantly lower than the measured values for all the alloys. The model also fails to capture the substantial jump in the orbital moment with the addition of Co at low concentrations $(<10 \%)$, and the similarly precipitous drop as the alloy approaches pure Co.

\section{SUMMARY}

We present a comprehensive study on the crystalline structure, effective magnetization, saturation magnetization, perpendicular anisotropy, $g$ factor, and orbital magnetic moment for 10-nm-thick binary alloys $\mathrm{Ni}_{x} \mathrm{Co}_{1-x}, \mathrm{Ni}_{x} \mathrm{Fe}_{1-x}$, and $\mathrm{Co}_{x} \mathrm{Fe}_{1-x}$ over the full range of alloy compositions. The measured saturation magnetization is consistent with the Slater-Pauling behavior for bulk specimens. By measuring the effective magnetization via FMR and the saturation 
magnetization via SQUID magnetometry, we calculate the perpendicular magnetic anisotropy energy density for all compositions. For a subset of alloy concentrations, we determine the bulk and interfacial contributions to the perpendicular magnetic anisotropy. While the bulk anisotropy energy density shows no discernable trend with alloy concentration, the interfacial contribution also exhibits Slater-Pauling-like behavior, which implies a fixed amount of interfacial anisotropy energy per localized, uncompensated, $d$-band spin. The measured $g$ factor agrees well with previously published results for the pure elements. Furthermore, we determine the orbital magnetic moments for all the alloys. Many of our measured values for $\mu_{\mathrm{L}}$ are in good agreement with previous predictions that were obtained from $a b$ initio calculations.
[1] L Pauling, The nature of the interatomic forces in metals, Phys. Rev. 54, 899 (1938).

[2] J. C. Slater, Electronic structure of alloys, J. Appl. Phys. 8, 385 (1937).

[3] R. M. Bozorth, Ferromagnetism (IEEE, New York, 2003).

[4] Y. Kakehashi, Modern Theory of Magnetism in Metals and Alloys (Springer, Berlin, 2012).

[5] J. Kübler, Theory of Itinerant Electron Magnetism (Oxford University Press, New York, 2000).

[6] A. P. Malozemoff, A. R. Williams, and V. L. Moruzzi, Band-gap theory of strong ferromagnetism: Application to concentrated crystalline and amorphous Fe- and Co-metalloid alloys, Phys. Rev. B 29, 1620 (1984).

[7] A. Williams, V. Moruzzi, A. Malozemoff, and K. Terakura, Generalized Slater-Pauling curve for transition-metal magnets, IEEE Trans. Magn. 19, 1983 (1983).

[8] M. A. W. Schoen et al., Ultra-low magnetic damping of a metallic ferromagnet, Nat. Phys. 12, 839 (2016).

[9] Ultrathin Magnetic Structures I (Springer, Berlin, 1994).

[10] P. K. Amiri et al., Low write-energy magnetic tunnel junctions for high-speed spin-transfer-torque MRAM, Electron Device Lett. 32, 57 (2011).

[11] S. Kaka et al., Spin transfer switching of spin valve nanopillars using nanosecond pulsed currents, J. Magn. Magn. Mater. 286, 375 (2005)

[12] C. A. Bauer and P. E. Wigen, Spin-wave resonance studies in invar films, Phys. Rev. B 5, 4516 (1972).

[13] A. J. P. Meyer and G. Asch, Experimental $g^{\prime}$ and $g$ values of Fe, Co, Ni, and their alloys, J. Appl. Phys. 32, S330 (1961).

[14] R. A. Reck and D. L. Fry, Orbital and spin magnetization in Fe-Co, Fe-Ni, and Ni-Co, Phys. Rev. 184, 492 (1969).

[15] F. El Gabaly et al., Imaging Spin-Reorientation Transitions in Consecutive Atomic Co Layers on Ru(0001), Phys. Rev. Lett. 96, 147202 (2006).

[16] M. Farle, W. Platow, A. N. Anisimov, P. Poulopoulos, and K. Baberschke, Anomalous reorientation phase transition of the magnetization in fet Ni/Cu(001), Phys. Rev. B 56, 5100 (1997).

[17] J. Prokop, D. A. Valdaitsev, A. Kukunin, M. Pratzer, G. Schönhense, and H. J. Elmers, Strain-induced magnetic anisotropies in Co films on Mo(110), Phys. Rev. B 70, 184423 (2004).

[18] C. Rizal, P. Gyawali, I. Kshattry, and R. K. Pokharel, Straininduced magnetoresistance and magnetic anisotropy properties of $\mathrm{Co} / \mathrm{Cu}$ multilayers, J. Appl. Phys. 111, $07 \mathrm{C} 107$ (2012).

[19] D Sander, The correlation between mechanical stress and magnetic anisotropy in ultrathin films, Rep. Prog. Phys. 62, 809 (1999).

[20] C. M. Schneider, P. Bressler, P. Schuster, J. Kirschner, J. J. de Miguel, and R. Miranda, Curie Temperature of Ultrathin Films of fcc-Cobalt Epitaxially Grown on Atomically Flat $\mathrm{Cu}(100)$ Surfaces, Phys. Rev. Lett. 64, 1059 (1990).

[21] L. Sun, P. C. Searson, and C. L. Chien, Finite-size effects in nickel nanowire arrays, Phys. Rev. B 61, R6463 (2000).

[22] A. García-Arribas et al., Tailoring the magnetic anisotropy of thin film permalloy microstrips by combined shape and induced anisotropies, Europ. Phys. J. B 86, 1 (2013).

[23] G. Bayreuther, M. Dumm, B. Uhl, R. Meier, and W Kipferl, Magnetocrystalline volume and interface anisotropies in epitaxial films: Universal relation and Néel's model (invited), J. Appl. Phys. 93, 8230 (2003).

[24] G. Bayreuther and G Lugert, Magnetization of ultra-thin epitaxial Fe films, J. Magn. Magn. Mater. 35, 50 (1983).

[25] M. Dumm et al., Magnetism of ultrathin FeCo (001) films on GaAs(001), J. Appl. Phys. 87, 5457 (2000).

[26] B. Heinrich et al., Development of magnetic anisotropies in ultrathin epitaxial films of $\mathrm{Fe}(001)$ and $\mathrm{Ni}(001)$, Appl. Phys. A 49, 473 (1989).

[27] F. Huang, M. T. Kief, G. J. Mankey, and R. F. Willis, Magnetism in the few-monolayers limit: A surface magneto-optic Kerreffect study of the magnetic behavior of ultrathin films of Co, $\mathrm{Ni}$, and Co-Ni alloys on $\mathrm{Cu}(100)$ and $\mathrm{Cu}(111)$, Phys. Rev. B 49 , 3962 (1994).

[28] E. Reiger et al., Magnetic moments and anisotropies in ultrathin epitaxial Fe films on ZnSe(001), J. Appl. Phys. 87, 5923 (2000).

[29] H. T. Nembach, T. J. Silva, J. M. Shaw, M. L. Schneider, M. J. Carey, S. Maat, and J. R. Childress, Perpendicular ferromagnetic resonance measurements of damping and Landé $g$-factor in sputtered $\left(\mathrm{Co}_{2} \mathrm{Mn}_{1-x} \mathrm{Ge}_{x}\right.$ films, Phys. Rev. B 84, 054424 (2011).

[30] J. M. Shaw, H. T. Nembach, T. J. Silva, and C. T. Boone, Precise determination of the spectroscopic $g$-factor by use of broadband ferromagnetic resonance spectroscopy, J. Appl. Phys. 114, 243906 (2013).

[31] M. T. Kief and W. F. Egelhoff, Growth and structure of Fe and Co thin films on $\mathrm{Cu}(111), \mathrm{Cu}(100)$, and $\mathrm{Cu}(110)$ : A comprehensive study of metastable film growth, Phys. Rev. B 47, 10785 (1993).

[32] J. Pelzl et al., Spin-orbit-coupling effects on $g$-value and damping factor of the ferromagnetic resonance in $\mathrm{Co}$ and $\mathrm{Fe}$ films, J. Phys.: Condens. Matter. 15, S451 (2003).

[33] M. Tischer, O. Hjortstam, D. Arvanitis, J. H. Dunn, F. May, K. Baberschke, J. Trygg, J. M. Wills, B. Johansson, and O. Eriksson, Enhancement of Orbital Magnetism at Surfaces: Co on $\mathrm{Cu}(100)$, Phys. Rev. Lett. 75, 1602 (1995).

[34] C.-H. Lambert et al., Quantifying perpendicular magnetic anisotropy at the $\mathrm{Fe}-\mathrm{MgO}(001)$ interface, Appl. Phys. Lett. 102, 122410 (2013).

[35] D. Marko, T. Strache, K. Lenz, J. Fassbender, and R Kaltofen, Determination of the saturation magnetization of ion irradiated 
Py/Ta samples using polar magneto-optical Kerr effect and ferromagnetic resonance, Appl. Phys. Lett. 96, 022503 (2010).

[36] A. H. Morrish, The Physical Principles of Magnetism (IEEE, New York, 2001).

[37] P. Bruno, Magnetic surface anisotropy of cobalt and surface roughness effects within Neel's model, J. Phys. F: Met. Phys. 18, 1291 (1988).

[38] L Neel, L'approche á la saturation de la magnétostriction, J. Phys. Radium 15, 376 (1954).

[39] P. Bruno and J.-P. Renard, Magnetic surface anisotropy of transition metal ultra thin films, Appl. Phys. A 49, 499 (1989).

[40] M. T. Johnson, P. J. H. Bloemen, F. J. A. den Broeder, and J. J. de Vries, Magnetic anisotropy in metallic multilayers, Rep. Prog. Phys. 59, 1409 (1996).

[41] J. M. Shaw, H. T. Nembach, and T. J. Silva, Damping phenomena in $\mathrm{Co}_{90} \mathrm{Fe}_{10} / \mathrm{Ni}$ multilayers and alloys, Appl. Phys. Lett. 99, 012503 (2011).

[42] M. Watanabe, M. Homma, and T. Masumoto, International conference on magnetism (part II) perpendicularly magnetized Fe-Pt ( $\left(\begin{array}{lll}0 & 0 & 1\end{array}\right)$ thin films with $(\mathrm{B} \bullet \mathrm{H})_{\max }$ exceeding $30 \mathrm{MG}$ Oe, J. Magn. Magn. Mater. 177, 1231 (1998).

[43] M. Farle, B. Mirwald-Schulz, A. N. Anisimov, W. Platow, and K. Baberschke, Higher-order magnetic anisotropies and the nature of the spin-reorientation transition in face-centered-tetragonal $\mathrm{Ni}(001) / \mathrm{Cu}(001)$, Phys. Rev. B 55, 3708 (1997).

[44] Ultrathin Magnetic Structures II (Springer, Berlin, 1994).

[45] J. M. Shaw, H. T. Nembach, and T. J. Silva, Measurement of orbital asymmetry and strain in $\mathrm{Co}_{90} \mathrm{Fe}_{10} / \mathrm{Ni}$ multilayers and alloys: Origins of perpendicular anisotropy, Phys. Rev. B 87, 054416 (2013).

[46] B. Újfalussy, L. Szunyogh, and P Weinberger, Magnetic anisotropy in $\mathrm{Fe} / \mathrm{Cu}(001)$ overlayers and interlayers: The highmoment ferromagnetic phase, Phys. Rev. B 54, 9883 (1996).

[47] A. Nilsson, J. Stöhr, T. Wiell, M. Aldén, P. Bennich, N. Wassdahl, M. G. Samant, S. S. P. Parkin, N. Mårtensson, J. Nordgren, B. Johansson, and H. L. Skriver, Determination of the electronic density of states near buried interfaces: Application to Co/Cu multilayers, Phys. Rev. B 54, 2917 (1996).

[48] R. Meservey and P. M. Tedrow, Spin-polarized electron tunneling, Phys. Rep. 238, 173 (1994).

[49] K. N. Altmann, D. Y. Petrovykh, G. J. Mankey, N. Shannon, N. Gilman, M. Hochstrasser, R. F. Willis, and F. J. Himpsel, Enhanced spin polarization of conduction electrons in $\mathrm{Ni}$ explained by comparison with $\mathrm{Cu}$, Phys. Rev. B 61, 15661 (2000).

[50] C. Carbone, M. Veronese, P. Moras, S. Gardonio, C. Grazioli, P. H. Zhou, O. Rader, A. Varykhalov, C. Krull, T. Balashov, A. Mugarza, P. Gambardella, S. Lebègue, O. Eriksson, M. I. Katsnelson, and A. I. Lichtenstein, Correlated Electrons Step by Step: Itinerant-to-Localized Transition of Fe Impurities in Free-Electron Metal Hosts, Phys. Rev. Lett. 104, 117601 (2010).

[51] R. Knut et al., Localization of Fe $d$-states in Ni-Fe-Cu alloys and implications for ultrafast demagnetization, arXiv:1508.03015 2015.

[52] B. N. Engel, C. D. England, R. A. Van Leeuwen, M. H Wiedmann, and C. M. Falco, Interface Magnetic Anisotropy in Epitaxial Superlattices, Phys. Rev. Lett. 67, 1910 (1991).

[53] T. Maeda, H. Yamauchi, and H. Watanabe, Spin wave resonance and exchange parameters in fcc Fe-Ni alloys, J. Phys. Soc. Jpn. 35, 1635 (1973).

[54] A. Malashevich, I. Souza, S. Coh, and D Vanderbilt, Theory of orbital magnetoelectric response, New J. Phys. 12, 053032 (2010).

[55] P. Söderlind, O. Eriksson, B. Johansson, R. C. Albers, and A. M. Boring, Spin and orbital magnetism in $\mathrm{Fe}-\mathrm{Co}$ and $\mathrm{Co}-\mathrm{Ni}$ alloys, Phys. Rev. B 45, 12911 (1992).

[56] S. Chadov et al., Orbital magnetism in transition metal systems: The role of local correlation effects, Europhys. Lett. 82, 37001 (2008).

[57] C Kittel, On the gyromagnetic ratio and spectroscopic splitting factor of ferromagnetic substances, Phys. Rev. 76, 743 (1949). 